Fetal Diagnosis and Therapy
Fetal Diagn Ther 2020;47:165-170

DOI: $10.1159 / 000500858$
Received: October 8, 2018

Accepted after revision: April 9, 2019 Published online: August 21, 2019

\title{
Neonatal Outcomes and Maternal Characteristics in Monochorionic Diamniotic Twin Pregnancies: Uncomplicated versus Twin-to-Twin Transfusion Syndrome Survivors after Fetoscopic Laser Surgery
}

\author{
Ciprian P. Gheorghe ${ }^{\mathrm{a}}$ Noemi Boring ${ }^{\mathrm{a}}$ Lovepreet Mann $^{\mathrm{a}}$ Roopali Donepudi ${ }^{\mathrm{a}}$ \\ Suzanne M. Lopez ${ }^{c}$ Suneet P. Chauhan ${ }^{b}$ Vineet Bhandari ${ }^{d}$ Kenneth J. Moise Jr. ${ }^{a}$ \\ Anthony Johnson ${ }^{\text {a }}$ Ramesha Papanna ${ }^{a}$ \\ a The Fetal Center, Children's Memorial Hermann Hospital, Houston, TX, USA; ' Department of Obstetrics, Gynecology \\ and Reproductive Sciences, McGovern Medical School - UTHealth, Houston, TX, USA; 'Department of Pediatrics, \\ McGovern Medical School - UTHealth, Houston, TX, USA; d Department of Pediatrics, Drexel University College of \\ Medicine, Philadelphia, PA, USA
}

\section{Keywords}

Fetal surgery $\cdot$ Perinatal outcome $\cdot$ Twin-to-twin transfusion syndrome

\begin{abstract}
Preterm birth remains a major complication of fetal laser surgery (FLS) due to twin-to-twin transfusion syndrome (TTTS). Objectives: We tested the hypothesis that neonatal outcomes in fetuses born at $>24$ weeks are worse in TTTS survivors compared to uncomplicated monochorionic diamniotic (MCDA) twins. Methods: 196 patients with TTTS treated with laser therapy and 91 uncomplicated MCDA gestations were compared. Neonatal outcomes included respiratory distress syndrome (RDS), transient tachypnea of the newborn (TTN), bronchopulmonary dysplasia, intraventricular hemorrhage, necrotizing enterocolitis, and neonatal death. Risk factors assessed were TTTS, maternal age, maternal body mass index, race, premature prolonged rupture of membranes, stage of TTTS, parity, and gestational age (GA)
\end{abstract}

at delivery. Results: GA at delivery was lower in the TTTS group ( $31.0 \pm 4.6$ vs. $33.5 \pm 2.4$ weeks, $p<0.001)$. RDS and TTN occurred at higher rates in the TTTS than in the uncomplicated MCDA group. After multivariate logistic regression, the only factor significantly associated with the composite adverse outcome was GA at delivery (OR $0.61 ; 95 \% \mathrm{Cl}$ : 0.58 0.7). Conclusion: TTTS twins treated with FLS are deliver 2.5 weeks earlier than uncomplicated MCDA twins. Respiratory complications were significantly higher in TTS twins and were mainly the consequence of the early GA at delivery.

(c) 2019 S. Karger AG, Basel

\section{Introduction}

Twin-to-twin transfusion syndrome (TTTS) affects approximately $10-15 \%$ of all monochorionic diamniotic (MCDA) twin pregnancies [1]. Left untreated, when it develops in the second trimester, it results in high rates of perinatal mortality and morbidity [2]. Furthermore, sur-

\section{KARGER}

(c) 2019 S. Karger AG, Basel

E-Mail karger@karger.com

www.karger.com/fdt
Ramesha Papanna, MD, MPH

The Fetal Center, Children's Memorial Hermann Hospital

6431 Fannin Street, Suite 3.274

Houston, TX 77030 (USA)

E-Mail ramesha.papanna@uth.tmc.edu 
vivors experience high rates of neurologic complications $[3,4]$. Several treatment strategies have been proposed, including selective fetal reduction, serial amnioreduction, and fetal laser surgery (FLS). FLS has been shown to be superior to serial amnioreduction and has become the standard treatment for TTTS [5]. Despite the success of FLS, several fundamental issues remain, including postoperative fetal loss and high rates of preterm delivery. The combination of higher survival rates and post-procedure prematurity have shifted outcomes from mortality to morbidity. Long-term sequelae have been noted which include neurodevelopmental deficits, cardiac sequelae, renal morbidity, and ischemic limb injury [6].

Several confounding factors make it difficult to truly assess the impact of TTTS on neonatal outcomes. MCDA twins experience a higher rate of overall complications than dichorionic twins, even in the absence of TTTS. The high incidence of prematurity after FLS for TTTS has the potential to further increase the rate of neonatal complications [7]. Previous studies have either been descriptive, used dichorionic twins as a comparison group, or did not correct for the difference in gestational age at delivery in TTTS survivors [8]. There are currently no other studies comparing outcomes between uncomplicated and TTTS MCDA twins. Hence, it remains unknown if TTTS independently increases neonatal morbidity.

We sought to compare the neonatal outcomes in a large cohort of FLS-treated pregnancies with a cohort of MCDA twins that did not develop TTTS. The Trial of Progesterone in Twins and Triplets to Prevent Preterm Birth (STTARS) was a double-blind randomized controlled study that sought to determine if injections of 17 alpha-hydroxyprogesterone caproate could reduce rates of preterm delivery in twin and triplet pregnancies [9]. The study showed no effect of progesterone administration on rates of preterm delivery, but it did contain a large number of MCDA twins with detailed neonatal outcomes. These twins were followed with serial sonography and excluded from the study if they developed TTTS. The objective of our study was to compare maternal characteristics, perinatal outcomes, including neonatal complications, between TTTS patients undergoing FLS versus uncomplicated MCDA twin gestations.

\section{Materials and Methods}

This is a secondary analysis combining two databases: (1) a prospective cohort study of consecutive patients treated for TTTS with FLS from one fetal treatment center during the period of September 2012 to May 2015 and (2) prospective data from the
STTARS trial collected from 2004 to 2007. Inclusion criteria were: MCDA twin gestations affected by TTTS that underwent FLS at our center and MCDA pregnancies that did not develop TTTS in the STTARS trial. Exclusion criteria included triplet pregnancies, selective reduction due to failed FLS, and delivery before 24 weeks leading to neonatal demise.

Briefly, patients with TTTS who were referred to our Fetal Center underwent a comprehensive ultrasound examination performed by registered medical diagnostic sonographers supervised by experienced fetal medicine specialists. Quintero Stage I patients that were symptomatic for polyhydramnios and had a short cervix along with stage II-IV patients underwent surgical intervention. Fetoscopic laser ablation was performed as previously described through a single-port fetoscopy under either local anesthetic in conjunction with intravenous sedation or general anesthesia [10]. The placental vascular equator was identified, and a diode laser was used to ablate all vascular anastomoses using a Solomon technique. In some cases, amnio-infusion using Ringer's lactate solution was performed in order to improve visualization. There were 3 cases of repeat laser procedures performed in this cohort, and they were included in the analysis. Two cases of selective reduction for failed laser procedure were excluded from the analysis. All patients returned to their referring physicians for follow-up for the remainder of the pregnancy.

The medical records from the patient's delivery and the neonatal hospital records were reviewed. The following preoperative variables were collected: maternal demographics (age, parity, previous preterm deliveries, and race) and staging using Quintero criteria. Postoperative variables included premature prolonged rupture of membranes defined as clinical confirmation of rupture before 34 weeks' gestation, gestational age at delivery, birth weight, and live birth rate. Neonatal outcomes that were collected using identical criteria in both databases were: respiratory distress syndrome (RDS), intraventricular hemorrhage (IVH), bronchopulmonary dysplasia (BPD), transient tachypnea of the newborn (TTN), necrotizing enterocolitis (NEC), neonatal death (within 30 days of delivery), and composite neonatal morbidity (at least one complication occurred). RDS was defined as the neonate receiving oxygen therapy $\left(\mathrm{FiO}_{2} \geq 0.40\right)$ for $\geq 24 \mathrm{~h}$. IVH was defined by neonatal ultrasound (grade III or IV). BPD was defined as a requirement for supplemental oxygen at 36 weeks' corrected gestational age. TTN was defined as a requirement for oxygen therapy and/or mechanical ventilation or CPAP during the first $24 \mathrm{~h}$ of life in newborns weighing $>1,000 \mathrm{~g}$ when the criteria for RDS or BPD were not met. NEC was defined as either the presence of intramural air on abdominal $\mathrm{x}$-ray, perforation seen on abdominal $\mathrm{x}$-ray, clinical evidence as suggested by erythema and induration of the abdominal wall, intra-abdominal abscess formation, or stricture formation following an episode of suspected NEC. Sepsis was defined as a positive blood or urine culture or evidence of cardiovascular collapse.

These variables from our dataset and the STTARS clinical trial (clinicaltrials.gov trial number NCT00099164) were combined in a unified database and analyzed.

\section{Statistical Analysis}

A baseline comparison between the TTTS cohort and the uncomplicated MCDA twin cohort was performed, including maternal demographics, gestational age at delivery, birth weight, and
Gheorghe et al. 


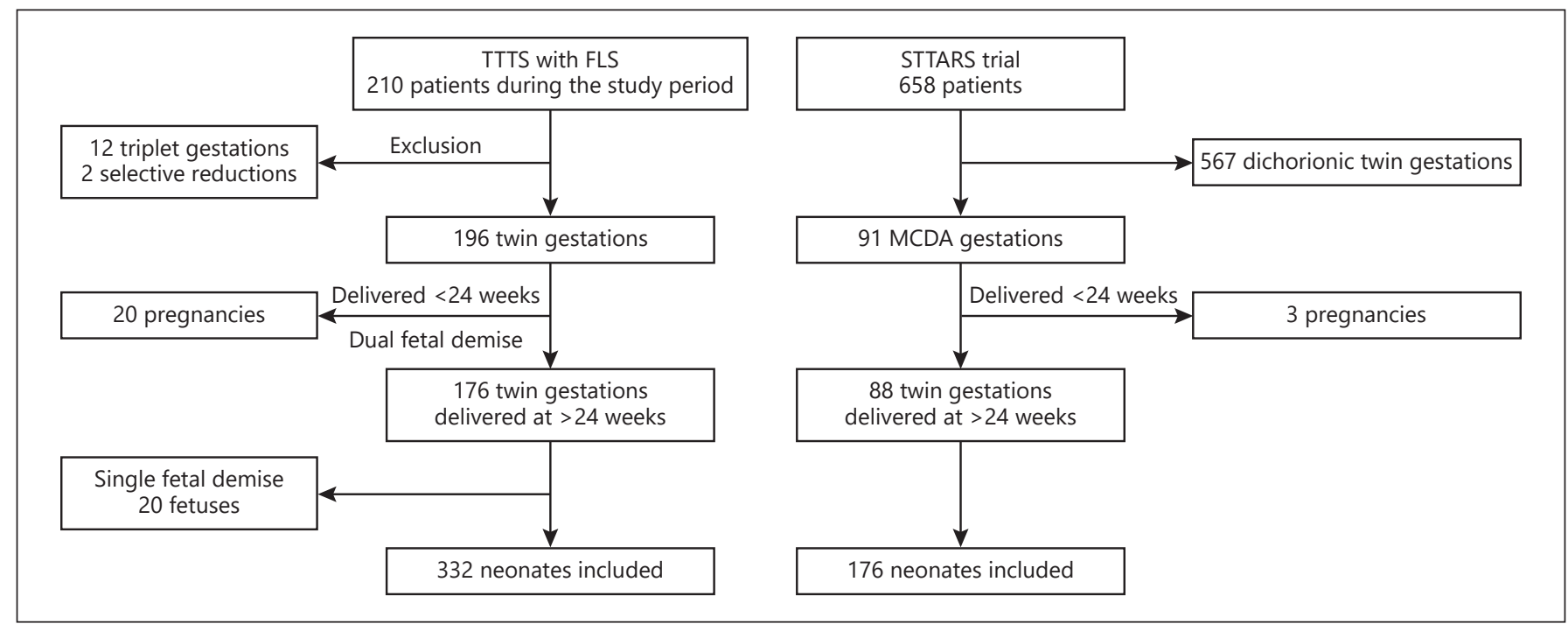

Fig. 1. Patient inclusion in the final cohort. TTTS, twin-to-twin transfusion syndrome; FLS, fetoscopic laser surgery; STTARS, Trial of Progesterone in Twins and Triplets to Prevent Preterm Birth; MCDA, monochorionic diamniotic.

neonatal complications. Descriptive statistics were used as appropriate for the distribution of each dataset.

Univariate analysis was performed to identify risk factors. $\mathrm{Pa}$ rameters were compared using the $\chi^{2}$ test for categorical variables; the Fisher exact test was used when an expected frequency was $<5$. An unpaired $t$ test was performed for continuous variables which satisfied the criteria for normal distribution by histogram, kurtosis, skewness, and P-P plots. If the data did not satisfy the criteria for normality, nonparametric analysis was performed. Subsequently, a multivariate logistic regression was performed from the risk factors that were found to be statistically significant $(p<0.05)$ to predict the composite outcome. Statistical significance was defined as a two-sided $p$ value $<0.05$ for all analyses. Pregnancies with in utero fetal demise before 24 weeks' gestation or delivered before 24 weeks were excluded from the analysis. The data for preoperative, intraoperative, and postoperative variables were extracted, and the analysis was performed using NCSS 11 software (NCSS LLC, Kaysville, UT, USA), and GraphPad Prism 5 (GraphPad, LaJolla, CA, USA).

\section{Results}

A total of 210 patients underwent FLS for TTTS during the study period. Twelve triplet pregnancies and two twin pregnancies who underwent selective reduction due to failed FLS were excluded. A total of 196 TTTS patients were included in the study. Of these, 20 (9.5\%) patients delivered before 24 weeks, and none of the neonates survived. A total of $91 \mathrm{MCDA}$ twins from the STTARS data who did not develop TTTS were included. Of these, 3 (3.3\%) patients delivered before
24 weeks, and none of the neonates survived. The difference did not reach statistical significance $(p=0.1)$. The clinical outcome of the two cohorts is illustrated in Figure 1.

Baseline characteristics of both groups are shown in Table 1 . The rate of a history of preterm labor was higher in the MCDA group; while parity, BMI, and the number of Caucasians were found to be higher in the TTTS group. In total, 176 out of 182 fetuses from the uncomplicated group and 332 out of 352 fetuses in the TTTS group survived until delivery and were included in the analysis. TTTS cases were delivered on average 2.5 weeks earlier than MCDA twins $(31.0 \pm 4.6$ vs. $33.5 \pm 2.4$ weeks; $p<$ 0.001 ). A Kaplan-Meier survival curve to assess gestational age at delivery illustrated a significant difference between the study groups, with TTTS pregnancies delivering at earlier gestational ages (Fig. 2; $p<0.01$ ). The Apgar score at $5 \mathrm{~min}$ and birth weight were lower in the TTTS group.

We compared neonatal outcomes between the groups, and the rates of IVH, BPD, NEC, and neonatal death were not statistically significant. The rates of RDS and TTN were higher in the TTTS group along with composite adverse outcomes ( $40 \%$ in the non-TTTS group vs. $58 \%$ in the TTTS group; $p<0.0001)$. The major contributor to the higher composite outcome was respiratory morbidity (Table 2). We then sought to determine which factors were driving this higher rate of morbidity. On logistic regression, we investigated all factors that were statistically 
Table 1. Patient characteristics of the study participants

\begin{tabular}{lccc}
\hline Patient characteristics & $\begin{array}{l}\text { Uncomplicated } \\
\text { MCDA }\end{array}$ & TTTS & p value \\
& 91 & 196 & - \\
\hline $\begin{array}{l}\text { Patients } \\
\text { Mean maternal age } \pm \text { SD, }\end{array}$ & $28.6 \pm 5.8$ & $28.3 \pm 6.6$ & 0.95 \\
$\quad$ years & $1(0-7)$ & $1(0-5)$ & $<0.0001$ \\
Median number of & $13(15 \%)$ & $8(4 \%)$ & $<0.001$ \\
$\quad$ parities (min.-max.) & & $28.9 \pm 6.2$ & 0.001 \\
History of preterm birth & $26.4 \pm 6.1$ & $170(86 \%)$ & $<0.0001$ \\
Mean BMI \pm SD & $57(62 \%)$ & $13(7 \%)$ & \\
Race & $17(19 \%)$ & $13(7 \%)$ & \\
$\quad$ White & $17(19 \%)$ & $33(17 \%)$ & 0.04 \\
$\quad$ African American & $25(27 \%)$ & & \\
$\quad$ Other & & & \\
$\quad$ Hispanic & & & \\
\hline
\end{tabular}

BMI, body mass index.

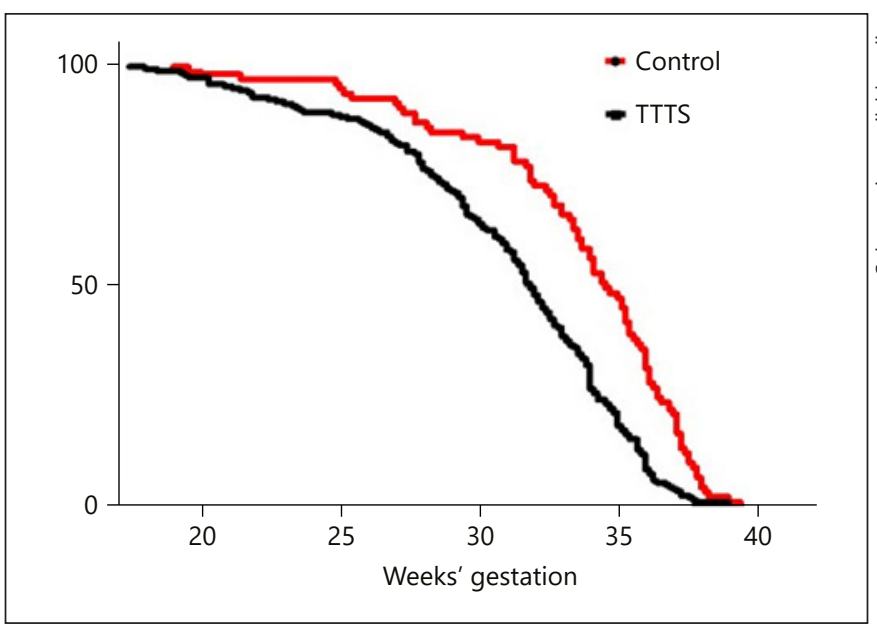

Fig. 2. Survival plot of gestational age at delivery. TTTS, twin-totwin transfusion syndrome.

significant $(p<0.05)$ between the two groups. Gestational age at delivery (OR 0.665; 95\% CI: 0.608-0.72) was the only factor significantly associated with the composite outcome (Fig. 3).

\section{Discussion}

The main finding of this study was the increased rate of respiratory neonatal morbidity which included RDS and TTN in the neonates with TTTS. This was mainly driven by gestational age at delivery, not due to the sec- ondary effects of TTTS. There were significant differences in maternal demographics between the two cohorts including BMI, race, ethnicity, and prior history of preterm birth. The average gestational age at delivery in the TTTS patients treated with laser surgery was 2.5 weeks lower than in uncomplicated MCDA twins, which resulted in a lower birth weight and Apgar score at $5 \mathrm{~min}$.

Gestational age at delivery in uncomplicated MCDA twins is lower than anticipated in dichorionic diamniotic twins (gestation of 35-36 weeks) [11]. However, in a recent study, uncomplicated MCDA twins without TTTS were delivered earlier than dichorionic twins, with $47 \%$ of all cases delivering prior to 34 weeks' gestation [12]. Our cohort of twins uncomplicated by TTTS were delivered relatively early at 33.5 weeks' gestation. MCDA pregnancies are at risk of other complications (selective growth restriction, twin anemia polycythemia sequence) that were unfortunately not captured by our study. Therefore, our control group was uncomplicated by TTTS but not necessarily uncomplicated by other morbidities unique to MCDA pregnancies. We noted a higher BMI in the TTTS group. The BMI was recorded at presentation for the treatment in the TTTS group and was likely increased due to polyhydramnios. To our knowledge, the BMI has not been previously implicated as a risk factor for TTTS but it is an observation deserving further investigation. The higher incidence of Caucasians in the TTTS group could point not only to a difference in demographics but also to a disparity in access to care. These differences raise important concerns that deserve to be further explored in follow-up studies.

We have shown that, in twin pregnancies successfully treated with FLS that survive to viability, the main determinant of neonatal complications is gestational age at delivery and less likely the disease itself. Other studies have shown that gestational age at delivery is also the main risk factor for poor long-term neurodevelopmental outcomes [13]. This underscores the importance of developing new strategies that reduce the high rates of preterm deliveries experienced by post-FLS patients. Several risk factors, some modifiable, are known to be associated with preterm delivery after the procedure. We have also recently shown that elective deliveries can account for up to $20 \%$ of these preterm deliveries after FLS treatment [14]. Our findings should encourage further studies aimed at reducing the high rate of postprocedural prematurity. It should also encourage providers to allow pregnancies to proceed as long as safely feasible following FLS. 
Table 2. Neonatal outcomes of patients delivered after 24 weeks

\begin{tabular}{lccc}
\hline Neonatal outcomes & Uncomplicated MCDA twins & TTTS twins after FLS & $p$ value \\
\hline Patients & $\mathbf{1 7 6}$ & 332 & $<0.001$ \\
Mean GA at delivery \pm SD, weeks & $33.5 \pm 4.2$ & $31.02 \pm 4.5$ & $<0.0001$ \\
Delivery <24 weeks & $3(3 \%)$ & $20(10 \%)$ & $<0.001$ \\
Median Apgar score at 5 min (range) & $9(1-9)$ & $1,581 \pm 716$ & 0.9 \\
Mean BW \pm SD, g & $2,016 \pm 674$ & $6(1.8 \%)$ & 0.9 \\
NEC & $3(1.7 \%)$ & $6(1.8 \%)$ & $<0.0001$ \\
Neonatal death & $3(1.7 \%)$ & $193(58 \%)$ & \\
Composite outcome & $71(40 \%)$ & \\
\hline \multicolumn{2}{l}{ Intrauterine fetal demises prior to viability are excluded from the analysis. } \\
BW, birth weight; GA, gestational age; NEC, necrotizing enterocolitis. \\
\hline
\end{tabular}

There are some limitations to our study. Since this was a combined cohort from two different studies, the groups had some differences at baseline. The cohorts were not collected simultaneously and from different centers; thus, they may have different characteristics. This is somewhat mitigated by the fact that both cohorts were collected at North American academic centers and have similar baseline characteristics. Since the groups were collected almost a decade apart from different centers, intra-institutional variability and changes in neonatal management undoubtedly introduced a level of variability that is difficult to account for. Unfortunately, there is currently no large cohort of uncomplicated MCDA twins to be used as a comparison. The great majority of MCDA twins we see at our center are referred when there is already concern for evolving TTTS, making the recruitment of a suitable uncomplicated cohort difficult. When possible, we adjusted for some of these limitations by including those factors in the logistic regression.

Our study was also not able to fully explore the full range of neonatal complications that can occur in the two groups since some of that data were not available to us. We did not have data on rates of retinopathy of prematurity or the incidence of other intracranial lesions such as white matter injury. Additionally, TTTS can lead to increased rates of cardiac disorders, limb injuries, renal and hematological sequelae. Unfortunately, this expanded set of outcomes was not captured by our database.

We also decided to exclude pregnancies delivered before 24 weeks from the logistic regression analysis. These pregnancies universally resulted in fetal or neonatal demise. This was undertaken for several reasons. We were

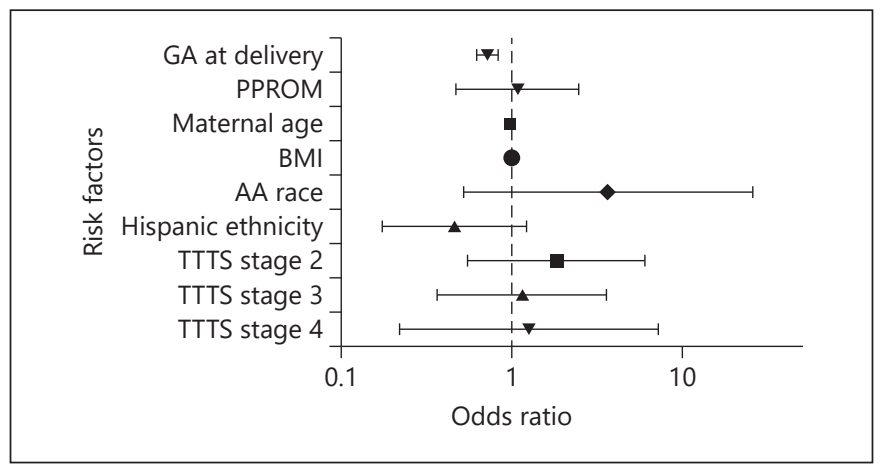

Fig. 3. Logistic regression model of risk factors contributing to neonatal morbidity. GA, gestational age; PPROM, premature prolonged rupture of membranes; BMI, body mass index; AA, African American; TTTS, twin-to-twin transfusion syndrome.

interested in determining any effects of TTTS in those fetuses that survived the periprocedural period and recovered successfully from the disease. Also, there is still considerable intra-institutional variability in the resuscitation practices of neonates delivered before 24 weeks' gestation. While more proactive and aggressive care for neonates born in the periviable period has increased survival rates [15], there were no survivors in our cohort. In a previous study, we have explored death rates and risk factors associated with demises in TTTS twins [16]. We were interested in comparing residual risk that can be attributed to TTTS in the fetuses that survived the procedure.

Our study guides providers in counseling parents on the survival and neonatal outcomes after laser therapy for TTTS. Our findings indicate that once viability has been achieved, neonatal outcomes will be equivalent to 
uncomplicated MCDA pregnancies of an equal gestational age. Our findings should also encourage future research efforts to reduce the rates of prematurity seen after fetal surgery. Our previous report indicated that spontaneous preterm birth occurs in over $60 \%$ of all post-laser procedure pregnancies [17]. Further studies are needed in order to explore interventions such as pessary and progesterone for short cervix at the time of procedure in order to improve gestational age at delivery.

\section{Statement of Ethics}

The study was approved by the Institutional Review Board of at the McGovern Medical School - UTHealth (IRB\# HSCMS-13-0712). The patients who were undergoing FLS were approached and consented for the collection of maternal and neonatal records prior to the procedure.

\section{Disclosure Statement}

The authors report no conflicts of interest.

\section{References}

1 Sebire NJ, Snijders RJ, Hughes K, Sepulveda $\mathrm{W}$, Nicolaides KH. The hidden mortality of monochorionic twin pregnancies. Br J Obstet Gynaecol. 1997 Oct;104(10):1203-7.

2 Habli M, Lim FY, Crombleholme T. Twin-totwin transfusion syndrome: a comprehensive update [x.]. Clin Perinatol. 2009 Jun;36(2): 391-416.

3 van Klink JM, Koopman HM, Rijken M, Middeldorp JM, Oepkes D, Lopriore E. Long-Term Neurodevelopmental Outcome in Survivors of Twin-to-Twin Transfusion Syndrome. Twin Res Hum Genet. 2016 Jun;19(3):255-61.

4 van Klink JM, Koopman HM, van Zwet EW, Middeldorp JM, Walther FJ, Oepkes D, et al. Improvement in neurodevelopmental outcome in survivors of twin-twin transfusion syndrome treated with laser surgery. Am J Obstet Gynecol. 2014 Jun;210(6):540.e1-7.

5 Senat MV, Deprest J, Boulvain M, Paupe A, Winer N, Ville Y. Endoscopic laser surgery versus serial amnioreduction for severe twinto-twin transfusion syndrome. N Engl J Med. 2004 Jul;351(2):136-44.

6 Lopriore E, Lewi L, Oepkes D, Debeer A, Vandenbussche FP, Deprest J, et al. In utero acquired limb ischemia in monochorionic twins with and without twin-to-twin transfusion syndrome. Prenat Diagn. 2008 Sep;28(9): $800-4$.
7 Papanna R. The problem of preterm delivery after laser surgery. Am J Perinatol. 2014 Sep; 31(S 01 Suppl 1):S47-50.

8 Lopriore E, Sueters M, Middeldorp JM, Oepkes D, Vandenbussche FP, Walther FJ. Neonatal outcome in twin-to-twin transfusion syndrome treated with fetoscopic laser occlusion of vascular anastomoses. J Pediatr. 2005 Nov; 147(5):597-602.

9 Rouse DJ, Caritis SN, Peaceman AM, Sciscione A, Thom EA, Spong CY, et al.; National Institute of Child Health and Human Development Maternal-Fetal Medicine Units Network. A trial of 17 alpha-hydroxyprogesterone caproate to prevent prematurity in twins. N Engl J Med. 2007 Aug;357(5):454-61.

10 Moise KJ Jr, Dorman K, Lamvu G, Saade GR, Fisk NM, Dickinson JE, et al. A randomized trial of amnioreduction versus septostomy in the treatment of twin-twin transfusion syndrome. Am J Obstet Gynecol. 2005 Sep;193(3 Pt 1):701-7.

11 Committee on Practice Bulletins-ObstetricsSociety for Maternal-Fetal Medicine. Practice Bulletin No. 169: Multifetal Gestations: Twin, Triplet, and Higher-Order Multifetal Pregnancies. Obstet Gynecol. 2016 Oct; 128(4):e131-46.

12 Coutinho Nunes F, Domingues AP, Vide Tavares $\mathrm{M}$, Belo A, Ferreira C, Fonseca E, et al.
Monochorionic versus dichorionic twins: are obstetric outcomes always different? J Obstet Gynaecol. 2016 Jul;36(5):598-601.

13 van Klink JM, Slaghekke F, Balestriero MA, Scelsa B, Introvini P, Rustico M, et al. Neurodevelopmental outcome at 2 years in twintwin transfusion syndrome survivors randomized for the Solomon trial. Am J Obstet Gynecol. 2016 Jan;214(1):113.e1-7.

14 Malshe A, Snowise S, Mann LK, Boring N, Johnson A, Bebbington MW, et al. Preterm delivery after fetoscopic laser surgery for twin-twin transfusion syndrome: etiology and its risk factors. Ultrasound Obstet Gynecol. 2017 May;49(5)612-6.

15 Backes CH, Rivera BK, Haque U, Srouji L, Beck S, Yin $\mathrm{H}$, et al. A Proactive Approach to Neonates Born at 23 Weeks of Gestation. Obstet Gynecol. 2015 Nov;126(5):939-46.

16 Snowise S, Moise KJ, Johnson A, Bebbington MW, Papanna R. Donor Death After Selective Fetoscopic Laser Surgery for Twin-Twin Transfusion Syndrome. Obstet Gynecol. 2015 Jul;126(1):74-80.

17 Malshe A, Snowise S, Mann LK, Boring N, Johnson A, Bebbington MW, et al. Preterm delivery after fetoscopic laser surgery for twin-twin transfusion syndrome: etiology and risk factors. Ultrasound Obstet Gynecol. 2017 May;49(5):612-6. 